\title{
Modeling Nonreactive Molecule-Surface Systems on Experimentally Relevant Time and Length Scales: Dynamics and Conductance of Polyfluorene on $\mathrm{Au}(111)$
}

\author{
Zhi Li, ${ }^{\dagger}$ Alexandre Tkatchenko, ${ }^{\ddagger}$ and Ignacio Franco ${ }^{* \dagger, \S_{(0)}}$ \\ ${ }^{\dagger}$ Department of Chemistry, University of Rochester, Rochester, New York 14611, United States \\ ${ }^{\ddagger}$ Physics and Materials Science Research Unit, University of Luxembourg, L-1511 Luxembourg, Luxembourg \\ ${ }^{\S}$ Department of Physics, University of Rochester, Rochester, New York 14611, United States
}

\section{Supporting Information}

\begin{abstract}
We propose a computationally efficient strategy to accurately model nonreactive molecule-surface interactions that adapts density functional theory calculations with the Tkatchenko-Scheffler scheme for van der Waals interactions into a simple classical force field. The resulting force field requires just two adjustable parameters per atom type that are needed to capture short-range and polarization interactions. The developed strategy allows for classical molecular dynamics simulation of molecules on surfaces with the accuracy of highlevel electronic structure methods but for system sizes $\left(10^{3}\right.$ to $10^{7}$ atoms) and timescales (picoseconds to microseconds) that go well beyond what can be achieved with first-principles methods. Parameters for $\mathrm{H}, \mathrm{sp}^{2} \mathrm{C}$, and $\mathrm{O}$ on $\mathrm{Au}(111)$ are developed and employed to atomistically model experiments that measure the conductance of a single polyfluorene on $\mathrm{Au}(111)$ as a continuous function of its length.

The simulations qualitatively capture both the gross and fine features of the observed conductance decay during initial junction elongation and lead to a revised atomistic understanding of the experiment.
\end{abstract}

$T^{\mathrm{k}}$ he interaction of organic materials and molecules with metal surfaces is of widespread interest to fundamental science and technology. ${ }^{1-4}$ The eventual control of the functionality of hybrid inorganic-organic systems has potential applications in a variety of fields including organic solar cells, photovoltaics, catalysis, surface science, nanotechnology, force spectroscopy, and molecular electronics. ${ }^{5-10}$

Recently, there have been significant advances in density functional theory (DFT) to describe inorganic-organic interfaces from first principles. ${ }^{11-13}$ Those advances have led to quantitative atomistic insights into the energetics and static properties of molecules on surfaces ${ }^{14,15}$ and to capturing dynamical events, usually in the femtosecond to picosecond timescale for systems with 10-100 atoms, that can be modeled using first-principles based molecular dynamics (MD) using present-day computational resources. ${ }^{16}$

Further progress in atomistic understanding of current experiments requires the ability to model metal/molecule dynamics for system sizes and timescales that are beyond the reach of DFT-based or ab initio techniques. This is required, for instance, to capture important conformational changes on the picosecond to millisecond timescale or to attempt to bridge the large gap (9-12 orders of magnitude) between STM/AFM experimental pulling speeds and their computational counterparts. ${ }^{17}$ For this, accurate and computationally inexpensive force fields (FFs) that can be employed in classical MD are needed that capture the full complexity of metal-molecule interactions at all relevant scales and separations.

Here we propose a strategy to develop an interatomic pairwise FF (vdW-FF) for nonreactive metal-molecule interactions that allows modeling systems with $10^{3}$ to $10^{7}$ atoms on timescales as long as microseconds with the accuracy of first-principles descriptions of surface-molecule interactions. As shown below, vdW-FF yields correct structures and energetics and offers an accuracy significantly beyond what can be achieved with state-of-the-art $\mathrm{FFs}^{18,19}$ or standard Lennard-Jones and Buckingham potentials. The essential idea is to decompose the metal-molecule interactions, $V=V_{\mathrm{sp}}+V_{\mathrm{img}}$ $+V_{\text {vdW }}$, into a short-range and polarization $V_{\text {sp }}$, an electrostatic $V_{\text {img, }}$ and a van der Waals $V_{\text {vdW }}$ component, and adapt into an effective FF the Tkatchenko-Scheffler (TS) scheme for vdW interactions. $^{20}$ More precisely, we adapt the $\mathrm{DFT}+\mathrm{vdW}{ }^{\text {surf }}$ scheme $^{21,22}$ that accounts for metal screening effects ${ }^{23,24}$ and accurately captures the long-range metal-molecule interactions, desirable properties that the FF inherits. Importantly, because TS $C_{6}$ coefficients can be obtained through DFT-based

Received: December 22, 2017

Accepted: February 13, 2018

Published: February 13, 2018 
computations on small molecular fragments, the vdW component of the FF does not require fitting, considerably simplifying its development. In fact, as discussed below, the vdW-FF requires only two adjustable parameters per atom type-metal pair needed to capture $V_{\text {sp }}$.

Below, we specify the components of the vdW-FF and develop a parameter set for $\mathrm{C}\left(\mathrm{sp}^{2}\right), \mathrm{O}\left(\mathrm{sp}^{2}\right)$, and $\mathrm{H}$ on $\mathrm{Au}(111)$ from $\mathrm{DFT}+\mathrm{vdW} \mathrm{W}^{\text {surf }}$ absorption curves of a family of planar molecules. The vdW-FF is used to model experiments ${ }^{25}$ that, through conductance measurements, probe the dynamics of a $28 \mathrm{~nm}$ oligofluorene on $\mathrm{Au}(111)$ as it is mechanically elongated using an STM tip. As shown, the vdW-FF MD trajectory can capture the experimentally observed conductance-distance profile including detailed features such as plateaus and spikes, and leads to a revised understanding of the atomistic dynamics responsible for the experimental observations.

Consider the different components to the interaction potential between a molecule $M$ and a surface $S$. The longrange $\mathrm{vdW}$ interaction in second-order perturbation theory is given by

$$
V_{\mathrm{vdW}}=-\sum_{A \in M} \sum_{B \in S} f_{d}^{\mathrm{vdW}}\left(R_{A B}\right) \frac{C_{6}^{A B}}{R_{A B}^{6}}
$$

where the label $A \in M$ runs over atoms in the molecule and $B$ $\in S$ runs over atoms in the surface. All of the coefficients that enter into this dominant contribution to the interaction potential can be determined from first-principles and require no adjustable parameters. Specifically, the $C_{6}^{A B}$ vdW coefficient for atom-pair $A-B$ separated by a distance $R_{A B}$ is given by ${ }^{26}$

$$
C_{6}^{A B}=\frac{2 C_{6}^{A A} C_{6}^{B B}}{\frac{\alpha_{0}^{B}}{\alpha_{0}^{A}} C_{6}^{A A}+\frac{\alpha_{0}^{A}}{\alpha_{0}^{B}} C_{6}^{B B}}
$$

where $\alpha_{0}^{i}$ is the static polarizability of atom $i$ and $C_{6}^{i i}$ is its homonuclear $C_{6}$ coefficient. The effective values of the $\alpha_{0}^{i}, C_{6}^{i i}$, and the vdW radius $R_{i}^{0}$ for atom $i$ in different molecular environments and hybridization states have been reported in ref 27 for molecules and in ref 21 for atoms in metals where screening effects need to be taken into account. In turn, the term $f_{d}^{\mathrm{d} d}\left(R_{A B}\right)=1 /\left[1+\exp \left(-d\left(R_{A B} /\left(s_{R} D_{A B}^{0}\right)-1\right)\right)\right]$ is a function that damps the $A-B$ vdW interaction when $R_{A B}$ is smaller than the sum of the vdW radii $D_{A B}^{0}=R_{A}^{0}+R_{B}^{0}$. The coefficients $d=20$ and $s_{R}=0.94$, which adjust the steepness of $f_{d}^{\mathrm{vdW}}\left(R_{A B}\right)$ and the onset of the vdW correction, are chosen as in DFT + vdW ${ }^{\text {surf }}{ }^{20}$

The electrostatic interactions between the molecule and a bulk metallic surface are modeled via the method of images ${ }^{28,29}$ and do not require adjustable parameters. On a metallic surface, the net electrostatic interaction between the molecule and its image is given by $V_{\text {img }}=\sum_{A \in M} \sum_{A^{\prime} \in M^{\prime}} \frac{q_{A^{\prime}} q_{A^{\prime}}}{8 \pi \epsilon_{0}} \frac{1}{R_{A A^{\prime}}}$, where $\epsilon_{0}$ is vacuum permittivity and $R_{A A^{\prime}}$ is the distance between the charge $q_{A}$ in the molecule $M$ and the charge $q_{A}^{\prime}$ in its mirror molecule $M^{\prime}$ (with charges $\left\{q_{A^{\prime}}\right\}$ of opposite sign) with respect to the surface plane of the metal. The partial charges $\left\{q_{A}\right\}$ are taken to be located on the atoms and computed using the RESP procedure $^{30}$ to reproduce the molecular electrostatic potential.

At short distances, when the electronic cloud of atoms in the surface and the molecule overlap, Pauli repulsion, short-range electron transfer, and polarization effects arise. ${ }^{31}$ To capture short-range effects and any remaining attractive interactions, we employ a Born-Mayer type potential ${ }^{32,33}$

$$
V_{\mathrm{sp}}=\sum_{A \in M} \sum_{B \in S}\left(\mathcal{A}_{1}^{A B} P\left(b_{A B} R_{A B}\right) \mathrm{e}^{-b_{A B} R_{A B}}-\frac{\mathcal{A}_{2}^{A B}}{R_{A B}^{6}}\right)
$$

for $R>R_{\text {cutoff }}$ and $V_{\text {sp }}\left(R_{\text {cutoff }}\right)$ otherwise. The coefficients governing the short-range repulsion $\left(\mathcal{A}_{1}^{\mathrm{AB}}>0\right)$ and the residual attraction $\left(\mathcal{A}_{2}^{\mathrm{AB}}>0\right)$ between molecule and metal are the only adjustable parameters in the FF and are chosen to be identical for atom pairs of the same type. The polynomial $P\left(b_{A B} R_{A B}\right)=\sum_{n=0}^{6}\left(b_{A B} R_{A B}\right)^{n} / n !$, is used to partially cancel the exponential decay for short distances. We use a Born-Mayer parameter $b_{A B}=-0.33\left(R_{A}^{0}+R_{B}^{0}\right)+8.298\left(\operatorname{dim}\left[b_{A B}\right]=\AA^{-1}\right)$, as determined in ref 27 from the universal correlation between atomic vdW radius and $b_{A B}$. The cutoff length guarantees that the potential does not become attractive for very short distances and is chosen to be the position where the potential is maximum. We have found the $R^{-6}$ term in the $V_{\mathrm{sp}}$ to be effective in capturing both short-range attraction due to molecule/metal density overlap as well as longer range electrostatic polarization effects captured by the DFT functional. From a polarization viewpoint, a $R^{-6}$ term corresponds to a permanent atomic dipole on the molecule interacting with an induced dipole in the surface. This term is independent of the vdW component of the FF because it is not damped by $f_{d}^{\mathrm{vdW}}\left(R_{A B}\right)$.

Parameters for $\mathrm{sp}^{2} \mathrm{C}, \mathrm{H}$, and $\mathrm{sp}^{2} \mathrm{O}$ were developed by leastsquares fitting of $\mathcal{A}_{1}$ and $\mathcal{A}_{2}$ in the vdW-FF to PBE+vdW ${ }^{\text {surf }}$ adsorption curves ${ }^{21,34}$ for three planar conjugated molecules (benzene, DIP, and PTCDA). The chemical structure of the molecules, their adsorption curves, and the developed FF are shown in Figure 1. In the FF development, we employed the same set of optimized geometries that were used in the DFT $+\mathrm{vdW}^{\text {surf }}$ computations. The $\mathrm{vdW}$ interaction was computed from eq 1 taking into account the mild dependence of the $C_{6}(R)=C_{6} f(R)$ coefficients for $\mathrm{C}$ and $\mathrm{H}$ on the atom-gold distance $R$, as reported in ref 22 . In the computations, the gold surface area and depth were extended until convergence of the $\mathrm{vdW}$ interactions. The resulting parameters and the vdW coefficients for the three relevant types of atom pairs $(\mathrm{C}-\mathrm{Au}$, $\mathrm{H}-\mathrm{Au}, \mathrm{O}-\mathrm{Au}$ ) are included in Tables $\mathrm{S} 1-\mathrm{S} 3$ of the Supporting Information.

As is evident in Figure 1, the vdW-FF quantitatively captures (within $\sim 0.05 \mathrm{eV}$ ) the adsorption curves of the three molecules at all relevant molecule-surface distances $(>2.5 \AA)$. The longrange vdW interactions (Figure 1A) are captured without the need of any adjustable parameters. The magnitudes of $\mathcal{A}_{1}$ and $\mathcal{A}_{2}$ that result from the fit reflect the strength of the repulsive and attractive interactions that cannot be captured by $V_{\mathrm{vdW}}$ and $V_{\text {img. }}$. Further note that the molecule-metal interaction is dominated by $V_{\mathrm{vdW}}$ and $V_{\mathrm{sp}}$, with $V_{\text {img }}$ being two orders or magnitude smaller (see Figures S1 and S2 in the Supporting Information). Remarkably, the simple form for $V_{\mathrm{sp}}$ can accurately reproduce binding energy curves for benzene, DIP, and PTCDA molecules simultaneously and, as shown in Figure S3, the adsorption energy of tilted benzene also.

By contrast, attempts to capture these three curves using standard Lennard-Jones (LJ) or Buckingham potentials with one or two adjustable parameters leads to errors in the predicted adsorption curves $\sim 1 \mathrm{eV}$ (see Figures S4-S6), indicating that new FF functional forms, like the one proposed 


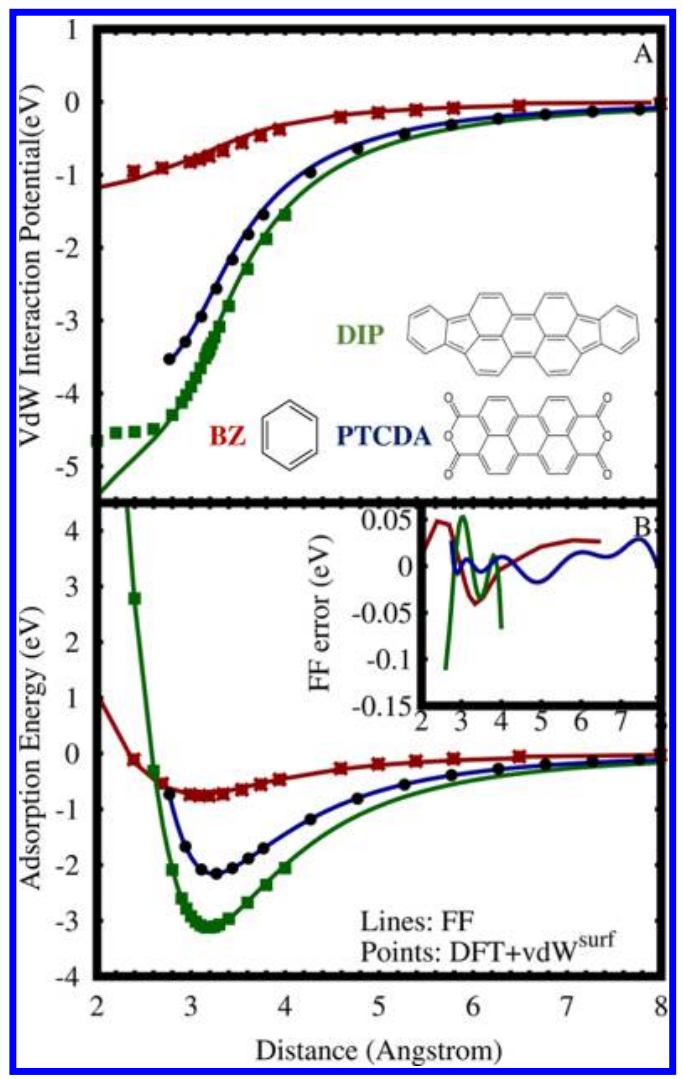

Figure 1. Force field (FF) for molecule-metal interactions. (A) vdWs and (B) net adsorption interactions for benzene (BZ), diindenoperylene (DIP), and perylene-3,4,9,10-tetracarboxylic dianhydrid (PTCDA) on $\mathrm{Au}(111)$ as a function of the molecule-surface distance. The Figure compares the FF (lines) with DFT $+\mathrm{vdW}^{\text {surf }}$ adsorption (dots) curves when the molecules are parallel to the surface. The inset shows the net FF errors.

here, are desirable to accurately capture metal-molecule interactions in both attractive and repulsive configurations. Furthermore, recently proposed $\mathrm{LJ}^{18}$ and ReaxFFs ${ }^{19}$ for metalmolecule interactions show significant deviations from the DFT $+v d W^{\text {surf }}$ adsorption curves (see Figure S7), demonstrating the need for more accurate FFs.

To illustrate the utility of the FF in a challenging setting, we modeled the experiments by $\mathrm{Grill}^{25}$ in which the conductance of a single conjugated polyfluorene PF (poly 9,9-dimethyl-9Hfluorene) is measured as a continuous function of its length. This is done by combining classical MD simulation of the pulling with Landauer transport computations of the conductance. $^{35}$ Modeling this class of experiments represents a major challenge because of the inherent disparity on timescales between theory and experiment, and the exponentially sensitivity of tunneling transport to changes in molecular conformation. The MD simulations employ the developed vdW-FF, as implemented in LAMMPS, ${ }^{36}$ and the OPLS FF ${ }^{37}$ for PF. For the $\mathrm{sp}^{3} \mathrm{C}$ in $\mathrm{PF}$ we employed the $\mathcal{A}_{i}^{\mathrm{AB}}(i=1,2)$ developed for $\mathrm{sp}^{2} \mathrm{C}$. The position of the Au atoms is kept fixed throughout the simulation. For numerical convenience, in the computations we employed a slightly simpler (and slightly less accurate with errors within $\sim 0.1 \mathrm{eV}$ ) form of the FF, where the spatial dependence of the $C_{6}$ coefficients is not taken into account. The employed parameters and fit are included in Table S3 and Figure S2. As shown later, even for this simplified
FF, the interaction potential accurately captures all relevant molecular events.

The chemical structure of PF and the general experimental setup is shown in Figure 2. The experiments take a PF of a

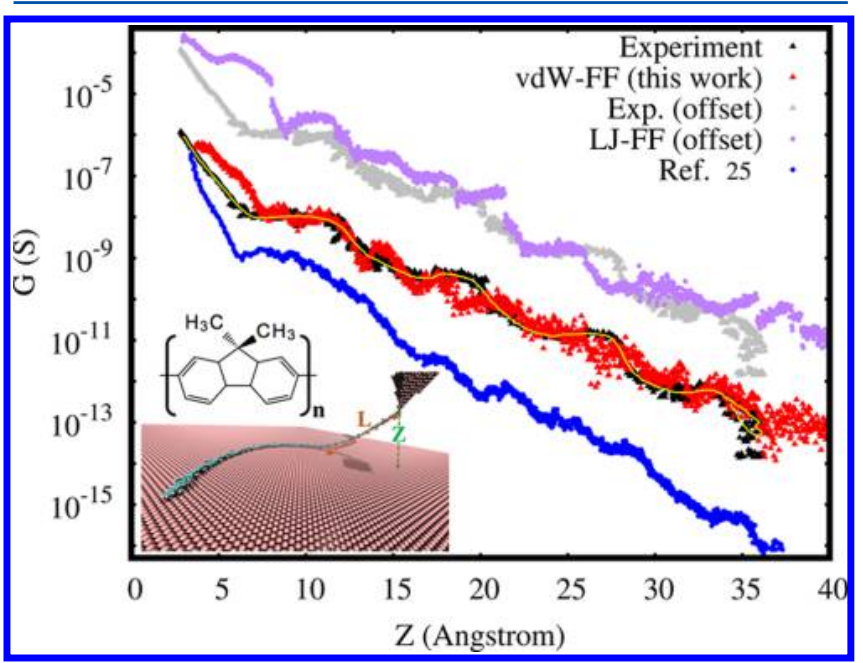

Figure 2. Comparison of experimental (black) and simulated (red) conductance curves as a single polyfluorene (PF) is pulled from a $\mathrm{Au}(111)$ surface by a STM. Here $Z$ is the STM tip-surface distance, which is experimentally controlled. The yellow line serves as a visual guide of the basic conductance pattern observed in experiments. Blue dots: Simulations in ref 25 based on minimizing the energy using a semiempirical Hamiltonian. Purple dots: Simulations using a LennardJones potential with parameters reported in ref 18 (simulation and experimental data are offset for clarity).

given length deposited on a $\mathrm{Au}(111)$ surface in vacuum at $10 \mathrm{~K}$ and create a bond between the end of the chain and a gold STM tip. Subsequently, the molecule is pulled at a constant speed while applying a voltage and measuring the current through the system. To model the experiment, we consider a $26.8 \mathrm{~nm}$ PF composed of 30 fluorene units on a $(130 \times 130 \times$ 2) flat $\mathrm{Au}(111)$ surface. This chain length is comparable to the ones employed in the experiment, and the surface is large compared with the molecule which ensures the convergence of the adsorption energy with surface size. As in the experiment, low-energy starting structures were obtained via 300 to $10 \mathrm{~K}$ thermal annealing. During the pulling, one terminal carbon of the PF was pulled with a constant speed of $10^{-7} \AA / \mathrm{fs}$ in the NVT ensemble $(10 \mathrm{~K})$ using a Langevin thermostat. At this pulling speed, the MD trajectory is largely insensitive to the initial (random) thermal velocities (see Figure S8). To mimic the elasticity of the gold STM tip, we used a $8 \mathrm{~N} / \mathrm{m}$ spring constant for the pulling device in silico along the pulling coordinate $Z$ and rigid in perpendicular directions. The oligomer was pulled for $40 \AA$, resulting in a $0.4 \mu$ s trajectory.

Electron transport is assumed to occur in the coherent tunneling regime in which the bias-dependent current $I(V)$ can be calculated from the Landauer formula

$$
I(V)=\frac{2 e}{h} \int_{-\infty}^{\infty} \mathrm{d} E\left[f_{\mathrm{L}}(E, V)-f_{\mathrm{R}}(E, V)\right] T(E, V)
$$

Here $e$ is the electron charge, $h$ is Planck's constant, $f_{\mathrm{L}, \mathrm{R}}$ are the Fermi functions of the left and right electrodes, respectively, and $T(E, V)$ is the transmission function; the Fermi function as well as the transmission are dependent on the injection energy $E$ and the applied bias voltage $V$. We assume low biases for 
which $I \approx G V$, where $G=G_{0} T\left(E_{\mathrm{F}}\right)$ is the zero-bias conductance, $G_{0}=2 e^{2} / h$ is the quantum of conductance, and $T\left(E_{\mathrm{F}}\right)$ is the transmission at the electrodes' common Fermi energy. The quantity $G$ is calculated for snapshots of the system encountered during pulling by computing $T\left(E_{\mathrm{F}}\right)$ using Green's functions. While the density of states of the electrodes was considered in the wide band limit approximation, explicit gold electrodes are taken into account to define the electrodemolecule couplings. In the transport computations, the terminal carbon from which the pulling is performed is connected to a $(4 \times 4 \times 3)$ Au tip placed $2.0 \AA$ away from the carbon along $Z$, which corresponds to gold-carbon equilibrium length. ${ }^{38}$ The electronic structure of this junction is modeled using the extended Hückel formalism ${ }^{39,40}$ because it captures essential electronic couplings and enables the simulations of several thousands conformations at reasonable computational cost. In this method, the Fermi level of gold $E_{\mathrm{F}}$ is a modeling parameter that determines the slope of the overall conductance decay. ${ }^{41}$ Here we systematically investigated the effect of changing $E_{\mathrm{F}}$ in the $G-Z$ curves and found that a value of $0.29 \mathrm{eV}$ below the $\mathrm{Au}$ 6s orbital energy (for which transport is dominated by the HOMO orbital) best describes the overall experimental conductance decay (see Figure S9).

Figure 2 shows the experimental (black) and simulated (red) conductance as the PF is elongated for a particular trajectory. An MD movie of the process and Figure S10, where the experimental curve is rigidly displaced for clarity, are included in the Supporting Information. The MD yields a fictitious dynamics that samples the canonical distribution for each $Z$. Thus the average simulated conductance at each $Z$ is comparable to experiment, even when computationally the pulling occurs on a much shorter timescale. Experimentally, the conductance for some molecules exhibits characteristic plateaus as the tip-surface distance $Z$ is increased. Remarkably, for particular initial orientations of the molecule in the surface, the MD simulations with the developed FF can capture the overall magnitude of the conductance, the conductance decay during pulling, the first plateau, and many of the fine experimentally observed features. Specifically, the simulations capture the initial decay of the experimental conductance as $Z$ increases from 3.8 to $6.8 \AA$. Then, in both theory and experiment a plateau from 7.5 to $11.9 \AA$ is observed with the computational plateau being $\sim 0.9 \AA$ shorter. In that plateau there is a conductance spike at $Z \approx 9.2 \AA$ that is present in both theory and experiment. From 11.9 to $16.4 \AA$ there is a second region where the conductance decays with elongation, with a spike at $13.7 \AA$ that also appears in both theory and experiment. Then, a second plateau region emerges from 16.4 to $19.4 \AA$. Simulations of this plateau agree with the experimental one from 16.4 to $18.4 \AA$ but underestimate the value of the conductance by $2.2 \times$ $10^{-10} \mathrm{~S}$ in the $18.4 \AA$ to $19.4 \AA$ region. From 19.4 to $40.0 \AA$, simulations capture the overall conductance decay.

For theory and experiment to agree, it is necessary to correctly capture both the molecule-surface interactions and the dynamics. For example, a previous computational analysis (blue curve in Figure 2) based on minimizing the energy of the chain during the elongation using an extended Hückel Hamiltonian only partially captured the plateaus observed in the experiment. ${ }^{25}$ This is because the minimum energy trajectory depends on the minimization algorithm, while the experimentally observed dynamics depends on thermally activated events that can only be captured via thermal sampling methods such as MD. Similarly, we performed simulations of the experiment with the recently developed LJ-FF for metalmolecule interactions in inorganic nanoparticles in ref 18 (in purple) using the same starting structure and with a $E_{\mathrm{F}}$ chosen to best describe the experiment (see Figure S11). The noncovalent component of this particular FF was found to lead to abrupt events in the gold-molecule dynamics during the pulling that do not resemble the experimental observations because it overestimates binding (see Figure S7). The method developed here can describe many of the experimentally observed features because it accurately captures metalmolecule interactions and the thermal dynamics.

The eigenenergy of the frontier orbitals of the chain remains essentially constant during the puling, indicating that the observed changes in the conductance are due to changes in the molecule-surface couplings during pulling. In ref 25 , the conductance plateaus were assigned to sliding motions of the polymer during the elongation that lead to an increase in $Z$ while keeping the peeled portion of the molecule $L$ approximately constant. To test this intuitive assignment, we monitor the center of mass $(x y z)$ coordinates of the terminal four fluorene units (labeled 1-4) in the oligomer during elongation; see Figure 3. The chain terminal segment is initially

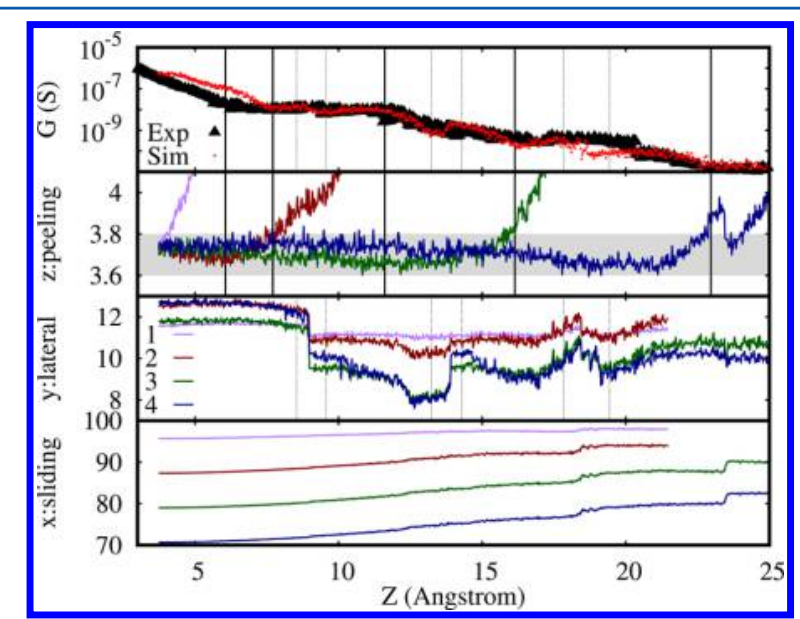

Figure 3. Peeling $(z)$, lateral $(y)$, and sliding $(x)$ motions of the center of mass of the four terminal fluorene units $(1-4)$ of the PF chain during the pulling. As a reference, the conductance-distance profile is included in the upper panel.

aligned along the $x$ direction, such that motion along $x$ indicates sliding, $y$ reflects lateral motions, and $z$ peeling. While sliding does occur during the pulling, there is no correlation between sliding and the emergence of conductance plateaus. Instead, the first plateau is seen to coincide with a situation, where unit 2 is being peeled beyond 3.8 A, while units 3 and 4 remain tightly attached to the surface keeping the contact, and thus the conductance, of the PF to the surface intact. The conductance decays are associated with peeling motion of the fluorene units in a subÅngstrom ( 3.6 to $3.8 \AA)$ range, highlighting the exponential sensitivity of the conductance to conformational changes. Furthermore, the simulations indicate that the fine spikes in the conductance near $Z=8 \AA$, $13 \AA$ observed both in theory and in experiment are due to abrupt lateral motion of the molecular wire on the surface. Such detailed atomistic understanding of the conformational dynamics can only be developed from a strong interplay between theory and experiment. 
The excellent agreement between theory and experiment highlights the power of the strategy to capture metal-molecule interactions using classical FFs. Naturally, differences can emerge between theory and experiment because the initial conformation, PF length, and orientation of the fluorene units along the chain in experiment and simulation do not necessarily coincide and can lead to differences in the conductance. Further simulation progress requires additional experimental input.

In conclusion, we have introduced a new, physically motivated, strategy to develop FFs for metal-molecule interactions that enables atomistically detailed simulation of the $\mathrm{MD}$ underlying surface science experiments, including STM, AFM, and novel tip-enhanced Raman, on experimentally relevant time and length scales with an accuracy beyond what can be achieved using standard Lennard-Jones or Buckingham potentials. The FF was employed to model and develop atomistic understanding of experiments that measure the conductance of a conjugated polymer as a continuous function of its length. The simulations qualitatively capture both the gross and fine features (plateaus, spikes, and regions of decay) of the observed conductance decay during junction elongation and show that the characteristic conductance plateaus do not necessarily arise due to sliding motion of the polymer. Instead, the decay in the conductance coincides with the initial peeling of a PF unit that is adsorbed on the surface. The plateaus coincide with peeling motion of a PF unit that no longer influences the electronic coupling between molecule and surface. The spikes coincide with lateral motions of the chain. The analysis further demonstrates that the TkatchenkoScheffler scheme for vdW can be adapted into useful force fields for metal-molecule interactions. This and related ${ }^{42}$ strategies can be employed to reduce parametrization efforts of new force-field developments.

\section{ASSOCIATED CONTENT}

\section{(S) Supporting Information}

The Supporting Information is available free of charge on the ACS Publications website at DOI: 10.1021/acs.jpclett.7b03389.

Additional details of the force field and the selection of the Fermi energy (PDF)

Movie of the MD trajectory (AVI)

\section{AUTHOR INFORMATION}

\section{Corresponding Author}

*E-mail: ignacio.franco@rochester.edu.

\section{ORCID}

Alexandre Tkatchenko: 0000-0002-1012-4854

Ignacio Franco: 0000-0002-0802-8185

\section{Notes}

The authors declare no competing financial interest.

\section{ACKNOWLEDGMENTS}

This work was supported by U. Rochester startup funds. We thank Fairoja Kabeer, Victor G. Ruiz, Wei Liu, and Nicola Ferri for helpful discussions.

\section{REFERENCES}

(1) Kronik, L.; Koch, N. Electronic Properties of Organic-Based Interfaces. MRS Bull. 2010, 35, 417-421.

(2) Koch, N. Organic Electronic Devices and Their Functional Interfaces. ChemPhysChem 2007, 8, 1438-1455.
(3) Tautz, F. S. Structure and Bonding of Large Aromatic Molecules on Noble Metal Surfaces: The Example of PTCDA. Prog. Surf. Sci. 2007, 82, 479-520.

(4) Rosei, F.; Schunack, M.; Naitoh, Y.; Jiang, P.; Gourdon, A.; Laegsgaard, E.; Stensgaard, I.; Joachim, C.; Besenbacher, F. Properties of Large Organic Molecules on Metal Surfaces. Prog. Surf. Sci. 2003, 71, 95-146.

(5) Pavliček, N.; Gross, L. Generation, Manipulation and Characterization of Molecules by Atomic Force Microscopy. Nat. Rev. Chem. 2017, $1,5$.

(6) Hybertsen, M. S.; Venkataraman, L. Structure-Property Relationships in Atomic-Scale Junctions: Histograms and Beyond. Acc. Chem. Res. 2016, 49, 452-460.

(7) Karakalos, S.; Xu, Y.; Cheenicode Kabeer, F.; Chen, W.; Rodríguez-Reyes, J. C. F.; Tkatchenko, A.; Kaxiras, E.; Madix, R. J.; Friend, C. M. Noncovalent Bonding Controls Selectivity in Heterogeneous Catalysis: Coupling Reactions on Gold. J. Am. Chem. Soc. 2016, 138, 15243-15250.

(8) Bergfield, J. P.; Ratner, M. A. Forty Years of Molecular Electronics: Non-Equilibrium Heat and Charge Transport at the Nanoscale. Phys. Status Solidi B 2013, 250, 2249-2266.

(9) Franco, I.; George, C. B.; Solomon, G. C.; Schatz, G. C.; Ratner, M. A. Mechanically Activated Molecular Switch through SingleMolecule Pulling. I. Am. Chem. Soc. 2011, 133, 2242-2249.

(10) Hoppe, H.; Sariciftci, N. S. Organic Solar Cells: An Overview. J. Mater. Res. 2004, 19, 1924-1945.

(11) Maurer, R. J.; Ruiz, V. G.; Camarillo-Cisneros, J.; Liu, W.; Ferri, N.; Reuter, K.; Tkatchenko, A. Adsorption Structures and Energetics of Molecules on Metal Surfaces: Bridging Experiment and Theory. Prog. Surf. Sci. 2016, 91, 72-100.

(12) Liu, W.; Tkatchenko, A.; Scheffler, M. Modeling Adsorption and Reactions of Organic Molecules at Metal Surfaces. Acc. Chem. Res. 2014, 47, 3369-3377.

(13) Tkatchenko, A.; Romaner, L.; Hofmann, O. T.; Zojer, E.; Ambrosch-Draxl, C.; Scheffler, M. Van der Waals Interactions Between Organic Adsorbates and at Organic/Inorganic Interfaces. MRS Bull. 2010, 35, 435-442.

(14) Wagner, C.; Fournier, N.; Tautz, F. S.; Temirov, R Measurement of the Binding Energies of the Organic-Metal Perylene-Teracarboxylic-Dianhydride/Au(111) Bonds by Molecular Manipulation Using an Atomic Force Microscope. Phys. Rev. Lett. 2012, 109, 076102.

(15) Aradhya, S. V.; Frei, M.; Hybertsen, M. S.; Venkataraman, L. Van Der Waals Interactions at Metal/Organic Interfaces at the SingleMolecule Level. Nat. Mater. 2012, 11, 872-876.

(16) Maurer, R. J.; Liu, W.; Poltavsky, I.; Stecher, T.; Oberhofer, H.; Reuter, K.; Tkatchenko, A. Thermal and Electronic Fluctuations of Flexible Adsorbed Molecules: Azobenzene on Ag (111). Phvs. Rev . Lett. 2016, 116, 146101.

(17) Franco, I.; Schatz, G. C.; Ratner, M. A. Single-Molecule Pulling and the Folding of Donor-Acceptor Oligorotaxanes: Phenomenology and Interpretation. J. Chem. Phys. 2009, 131, 124902.

(18) Pohjolainen, E.; Chen, X.; Malola, S.; Groenhof, G.; Häkkinen, H. A Unified AMBER-Compatible Molecular Mechanics Force Field for Thiolate-Protected Gold Nanoclusters. J. Chem. Theory Comput. 2016, 12, 1342-1350.

(19) Järvi, T. T.; Van Duin, A. C. T.; Nordlund, K.; Goddard, W. A. Development of Interatomic ReaxFF Potentials for Au-S-C-H Systems. I. Phvs. Chem. A 2011, 115, 10315-10322.

(20) Tkatchenko, A.; Scheffler, M. Accurate Molecular Van Der Waals Interactions from Ground-State Electron Density and FreeAtom Reference Data. Phys. Rev. Lett. 2009, 102, 073005.

(21) Ruiz, V. G.; Liu, W.; Zojer, E.; Scheffler, M.; Tkatchenko, A. Density-Functional Theory with Screened van der Waals Interactions for the Modeling of Hybrid Inorganic-Organic Systems. Phys. Rev. Lett. 2012, 108, 146103.

(22) Ruiz, V. G.; Liu, W.; Tkatchenko, A. Density-Functional Theory with Screened Van Der Waals Interactions Applied to Atomic and 
Molecular Adsorbates on Close-Packed and Non-Close-Packed Surfaces. Phys. Rev. B 2016, 93, 035118.

(23) Lifshitz, E. M. The Theory of Molecular Attractive Forces between Solids. JETP 1956, 2, 73.

(24) Zaremba, E.; Kohn, W. Van Der Waals Interaction Between an Atom and a Solid Surface. Phvs. Rev. B 1976, 13, 2270-2285.

(25) Lafferentz, L.; Ample, F.; Yu, H.; Hecht, S.; Joachim, C.; Grill, L. Conductance of a Single Conjugated Polymer as a Continuous Function of its Length. Science 2009, 323, 1193-1197.

(26) Stone, A. The Theory of Intermolecular Forces; OUP Oxford: Cambridge, U.K., 2013.

(27) Von Lilienfeld, O. A.; Tkatchenko, A. Two- and Three-Body Interatomic Dispersion Energy Contributions to Binding in Molecules and Solids. I. Chem. Phys. 2010, 132, 234109.

(28) Lang, N. D.; Kohn, W. Theory of Metal Surfaces: Induced Surface Charge and Image Potential. Phys. Rev. B 1973, 7, 3541-3550.

(29) Griffiths, D. Introduction to Electrodynamics; Prentice Hall: Upper Saddle River, NJ, 1999.

(30) Bayly, C. I.; Cieplak, P.; Cornell, W.; Kollman, P. A. A WellBehaved Electrostatic Potential based Method using Charge Restraints for Deriving Atomic Charges: The RESP Model. J. Phys. Chem. 1993, 97, 10269-10280.

(31) Van Vleet, M. J.; Misquitta, A. J.; Stone, A. J.; Schmidt, J. R. Beyond Born-Mayer: Improved Models for Short-Range Repulsion in ab Initio Force Fields. J. Chem. Theory Comput. 2016, 12, 3851-3870.

(32) Abrahamson, A. A. Born-Mayer-Type Interatomic Potential for Neutral Ground-State Atoms with $Z=2$ to $Z=105$. Phys. Rev. 1969, $178,76-79$.

(33) Buckingham, R. A. The Classical Equation of State of Gaseous Helium, Neon and Argon. Proc. R. Soc. London, Ser. A 1938, 168, 264283.

(34) Liu, W.; Carrasco, J.; Santra, B.; Michaelides, A.; Scheffler, M.; Tkatchenko, A. Benzene Adsorbed on Metals: Concerted Effect of Covalency and Van Der Waals Bonding. Phys. Rev. B 2012, 86, 245405.

(35) Carey, R.; Chen, L.; Gu, B.; Franco, I. When Can TimeDependent Currents be Reproduced by the Landauer Steady-State Approximation? I. Chem. Phvs. 2017, 146, 174101.

(36) Plimpton, S. Fast Parallel Algorithms for Short-Range Molecular Dynamics. J. Comput. Phvs. 1995, 117, 1-19.

(37) Jorgensen, W. L.; Maxwell, D. S.; Tirado-Rives, J. Development and Testing of the OPLS All-Atom Force Field on Conformational Energetics and Properties of Organic Liquids. J. Am. Chem. Soc. 1996, 118, 11225-11236.

(38) Benitez, D.; Shapiro, N. D.; Tkatchouk, E.; Wang, Y.; Goddard, W. A.; Toste, F. D. A Bonding Model for Gold(I) Carbene Complexes. Nat. Chem. 2009, 1, 482-486.

(39) Frisenda, R.; Janssen, V. A. E. C.; Grozema, F. C.; van der Zant, H. S. J.; Renaud, N. Mechanically Controlled Quantum Interference in Individual $\pi$-Stacked Dimers. Nat. Chem. 2016, 8, 1099-1104.

(40) Hutcheson, J.; Franco, I.; Nicolas, R.; Carignano, M.; Ratner, M. A.; Schatz, G. C. TRANSpull: Computes Pulling Coupled to Transport Properties of Single Molecules. nanoHUB 2011, DOI: 10.4231/D3ZS2KD79.

(41) Tomfohr, J. K.; Sankey, O. F. Complex Band Structure, Decay Lengths, and Fermi Level Alignment in Simple Molecular Electronic Systems. Phys. Rev. B 2002, 65, 245105.

(42) Cole, D. J.; Vilseck, J. Z.; Tirado-Rives, J.; Payne, M. C.; Jorgensen, W. L. Biomolecular Force Field Parameterization via Atoms-in-Molecule Electron Density Partitioning. J. Chem. Theory Comput. 2016, 12, 2312-2323. 